\title{
Index of Volume 11 (1991)
}

\section{Number 1}

Alfred M. Grundland and Bohdan Lawruk

The Lagrange-Riemann method for integration of quasilinear partial differential equations 1

\section{Gerd Herzog}

On universal functions and interpolation $\mathbf{2 1}$

\section{Rainer Brück}

Summability of sequences of polynomial interpolants in the roots of unity 27

George A. Anastassiou, Claudia Cottin and Heinz H. Gonska

Global smoothness of approximating functions $\mathbf{4 3}$

\section{J. A. Fridy and H. I. Miller}

A matrix characterization of statistical convergence $\mathbf{5 9}$

Tran Duc Van, Ha Huy Bang and R. Gorenflo

On Sobolev-Orlicz spaces of infinite order for a full Euclidean space 67

\section{Hans-Christoph Grungu}

The Dirichlet problem for some semilinear elliptic differential equations of arbitrary order $\mathbf{8 3}$

Arnold Knopfmacher and Lutz Lucht

The radius of convergence of power product expansions 91

\section{Number $2 / 3$}

\section{Simson Baron}

Summability factors with a rate and multipliers of double Fourier series of

Lipschitz classes 101

\section{Norbert Steinmetz}

Linear differential equations with exceptional fundamental sets 119

\section{Saverio Salemo and Antonio Vitolo}

$\mathrm{p}+2$ with few and bounded prime factors 129

\section{S. M. Mazhar}

Approximation by the partial sums of Fourier series 149

\section{Abhay K. Gaur and Zdislav V. Kovaifik}

Norms, states and numerical ranges on direct sums 155 


\section{Werner Varnhorn}

Zur Konstruktion einer numerischen Lösung des Anfangsrandwertproblems für die nichtlinearen Gleichungen von Navier - Stokes 191

Lyubomir Boyadjiev

On series in Laguerre and Hermite polynomials 209

Alfred M. Grundland and Peter J. Vassiliou

On the solvability of the Cauchy problem for Riemann double-waves by the MongeDarboux method 221

\section{Number 4}

\section{Johann Boos and Toivo Leiger}

Consistency theory for operator valued matrices 279

Árpád Elbert, Andrea Laforgia and Lee Lorch

Additional monotonicity properties of the zeros of Bessel functions 293

\section{A. K. Snyder and Gary S. Stoudt}

Basis in Banach space, strictly cosingular maps, and the Wilansky property 301

\section{Peter Köhler}

Dual approximation methods and Peano kernels 323

\section{Yoshihiro Mizuta}

Fine limits of potentials 345

Wolfram Jérôme Luther

Abelsche und Taubersche Sätze für Laguerre-Reihen $\mathbf{3 8 3}$

\section{Horst Alzer}

Corrigendum to: On an extension of Leibniz' product rule 395 